\title{
Article \\ Optimizing the Process Design of Oil-in-Water Nanoemulsion for Delivering Poorly Soluble Cannabidiol Oil
}

\author{
Agnieszka Lewińska
}

check for

updates

Citation: Lewińska, A. Optimizing the Process Design of Oil-in-Water Nanoemulsion for Delivering Poorly Soluble Cannabidiol Oil. Processes 2021, 9, 1180. https://doi.org/ $10.3390 /$ pr 9071180

Academic Editor: Urszula Bazylinska

Received: 28 May 2021

Accepted: 5 July 2021

Published: 7 July 2021

Publisher's Note: MDPI stays neutral with regard to jurisdictional claims in published maps and institutional affiliations.

Copyright: (C) 2021 by the author. Licensee MDPI, Basel, Switzerland. This article is an open access article distributed under the terms and conditions of the Creative Commons Attribution (CC BY) license (https:// creativecommons.org/licenses/by/ $4.0 /)$.
Faculty of Chemistry, University of Wroclaw, Joliot-Curie 14, 50-383 Wroclaw, Poland; agnieszka.lewinska@uwr.edu.pl or agnieszka.lewinska@chem.uni.wroc.pl; Tel.: +48-71-3757324

Keywords: nanoemulsion; CBD; surfactin; skin

\section{Introduction}

Cannabis (Cannabis sativa, L.) contains over 120 phytocannabinoids [1], but one of its main components is cannabidiol (CBD) (Figure 1). Despite its similar chemical structure to tetrahydrocannabinol, it does not exhibit psychotropic effects [2]. The legality of cannabis varies between countries but many permit medical use. Highly purified CBD Epidiolex $^{\circledR}$, a CBD oil preparation, was approved by the US FDA for the treatment of seizures associated with Lennox-Gastaut syndrome or Dravet syndrome, in June 2018. Since that time there has been a multitude of initial research studies indicating that CBD could offer a variety of potential therapeutic and wellness benefits. The most studied cannabinoids, tetrahydrocannabinol $(\triangle 9-\mathrm{THC})$ and $C B D$ have shown promising results in the management of chronic pain. They have also shown the capacity for limiting nausea induced by chemotherapy [3] anti-inflammation, along with demonstrating anticonvulsant and anxiolytic properties [4].

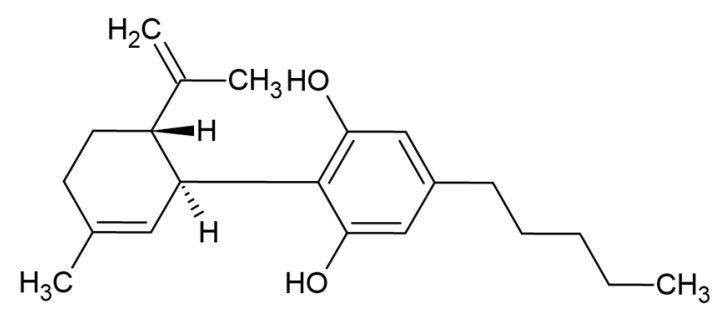

Figure 1. CBD structure. 
CBD has become increasingly popular in the wellness industry for its potential in relaxation and relieving inflammation $[5,6]$. Hemp seed oil is rich in omega- 3 and omega- 6 fatty acids and can therefore provide various nutrient benefits from consumption. It can also be used topically to relieve dry skin [7].

Research indicates that CBD receptors are also found in epidermal keratinocytes, cutaneous nerve fibers, dermal cells, melanocytes, eccrine sweat glands, and hair follicles [8-10]. Cutaneous biology is modulated by the human endocannabinoid system (ECS). Recent research has indicated that receptors from the ECS are presented in the skin playing a critical role in maintaining skin homeostasis and barrier function. Their dysregulation has been implicated in various skin disorders such as atopic dermatitis, itching, acne, hair growth, and hyper/hypopigmentation [11]. CBD is a highly lipophilic compound and it is usually supplied as an oil preparation. The lipophilic nature and consequent low aqueous solubility of cannabinoids, however, result in low bioavailability. Therefore, products based on CBD oils and creams designed for ingestion or topical use, have a relatively low bioavailability too. This presents a major difficulty in designing and systemically delivering cannabinoid-based formulations for most therapeutic applications. Research suggests that CBD oil may be beneficial for a many of different skin conditions. CBD contains many compounds with oil-reducing, anti-inflammatory, and antimicrobial properties that may help improve acne, which is the most common skin condition in humans. The study indicates that CBD can prevent sebocytes from creating too much sebum [12]. Additionally noted are the potential antibacterial and antifungal properties of the cannabis plant, which could help prevent acne due to infections on the skin [13]. The latest study notes that CBD may be useful for dryness and itching skin, helping soothe and reduce the appearance of irritation, so it may be useful for people with sensitive skin [14]. CBD also has antioxidant properties. Oxidative stress can contribute to the ageing process. Therefore, the antioxidant and anti-inflammatory properties of CBD may help prevent the appearance of ageing in the skin [15]. Since cannabinoids are delicate molecules, they are prone to degradation by oxidization, as well as by different enzymes, thus encapsulation could prevent their degradation and protect them from the unfavorable conditions of the external environment.

Technically, oil-in-water $(\mathrm{o} / \mathrm{w})$ emulsions are mixtures wherein water is the continuous phase and oil is the dispersed phase, often stabilized by one or more surfactants. These surfactants can be natural or artificial, and they are useful to reduce the surface molecular tension between oils and liquids. Nanoemulsions are an advanced part of emulsion nanotechnology and are usually produced by ultrasonic liquid processors that break the dispersed phase down into 10-500 nm droplets, which are much smaller than the range for conventional emulsions. They are kinetically stable colloidal delivery systems suitable for the protection, delivery, and encapsulation of active compounds. Nanoemulsion is being studied as an effective delivering tool for several diagnostic and therapeutic applications including structural design processes [16-18]. A previous study suggests also that nanoemulsions significantly increase the transdermal bioavailability of vitamin E and C [18]. To produce long-term stable $\mathrm{o} / \mathrm{w}$ nanoemulsions, it is necessary to use a suitable surfactant and technology. The type of carrier oils and not oils is a very important factor to create a physicochemical stable emulsion, since they may help to protect the emulsion against Ostwald ripening, which is the driving force that destabilizes an emulsion by droplet coarsening. The components of the formulation must remain compatible to create a stable system. The tendency to destabilize during storage through different mechanisms (Ostwald ripening, gravitational separation, coalescence, flocculation) necessitates the application of different surfactants to attain long-term stability. Studies demonstrated that commonly a mixture of surfactants performed better than single surface-active molecules $[19,20]$. With this in mind, we have designed and engineered a novel o/w nanoemulsion (Scheme 1) stabilized by a mixture of two surface-active agents: polyethylene glycol sorbitan monooleate and surfactin encapsulating the CBD oil. Polyethylene glycol sorbitan monooleate is a non-ionic surfactant and emulsifier often used in foods, cosmetics, and medications. The 
lipophilic group constitutes part of the structure is delivers exogenous oleic acid, which can directly incorporate itself into the cells and affects cell membrane properties [21].

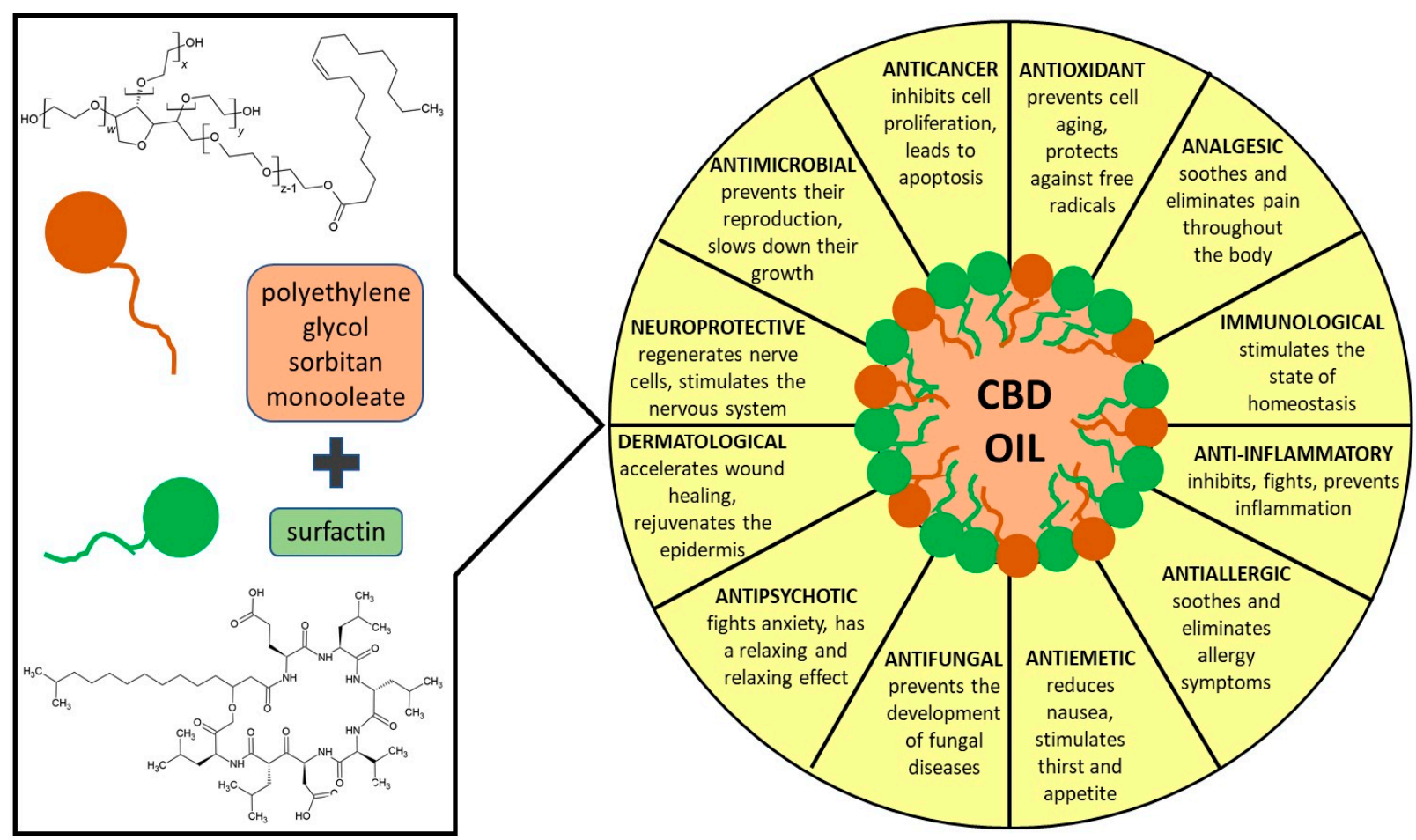

Scheme 1. General idea of the performed studies.

Surfactin is the cyclic lipopeptide biosurfactant produced by Bacillus subtilis with special properties-high surface activity, low toxicity, high biodegradability. The structure of the hydrophilic part consists of a peptide loop of seven amino acids (L-glutamic acid, L-leucine, D-leucine, L-valine, L-aspartic acid, D-leucine, and L-leucine) and a $\beta$-hydroxy fatty acid with a variable length carbon chain. The glutamic acid and aspartic acid residues give the ring its negative charge. The hydrophobic part consists of a fatty acid chain, which below critical micellar concentrations can extend freely into solution, and then participate in hydrophobic interactions. The structure of the hydrophilic part consists of a peptide loop of seven amino acids (L-glutamic acid, L-leucine, D-leucine, L-valine, L-aspartic acid, D-leucine, and L-leucine) and a $\beta$-hydroxy fatty acid of the variable length carbon chain. The glutamic acid and aspartic acid residues which constitute the ring carry their negative charge $(-2)$. The hydrophobic part represents a fatty acid chain, which below the critical micellar concentrations can participate in hydrophobic interactions, hence solution molecules maintain a characteristic "horse saddle" conformation that explains its large spectrum of biological activity. Surfactin exhibits a wide range of interactions with cell membranes, and besides its antifungal and antibacterial effect, it can also inhibit fibrin clot formation, and induce the formation of ion channels in lipid bilayer membranes, and has antitumor activities [22]. This surfactant is already successfully used in creating different nano-structures [23], including nanoemulsions [18,24,25].

In this study, we used surfactin ionic and polyethylene glycol sorbitan monooleate nonionic surfactant to emulsify CBD oil to design, fabricate and characterize self-assembly processes and creation of a nanoemulsion. CBD as an effective active compound with many beneficial effects on the skin was encapsulated in the o/w nanoemulsion. The preparation was carried out in two ways: with the sonication method and with a two-stage high-pressure homogenizer advanced homogenization (microfluidization). The results of the nanodroplets size, polydispersity, and Zeta potential have been confirmed by the DLS method. The compatibility and morphology of the obtained systems were imaged by 
TEM. The nanoemulsions were tested for biocompatibility with human skin cell lines, i.e., $\mathrm{HaCaT}$ keratinocytes and NHDF normal human dermal fibroblasts. In the last stage, we demonstrate the influence of the nanoemulsions on the condition of human skin: hydration and degree of discoloration by using an analyzer.

\section{Materials and Methods}

\subsection{Chemicals}

Polyethylene glycol sorbitan monooleate $(\mathrm{P})$ applied as a non-ionic surfactant was purchased from Sigma-Aldrich (Poznan, Poland). CBD oil (O) was a kind gift from the company Lessonia (Saint-Thonan, France), obtained by extracting hemp (Cannabis sativa) stems in a CBD oil base that contains the only composition of CBD without any THC content.

Surfactin (S) was obtained from Bacillus subtilis natto KB1 strains grown on rapeseed cake. Bacteria were grown on a modified Landy's medium. The preculture was carried out at $37^{\circ} \mathrm{C}$ with shaking at $180 \mathrm{rpm}$ medium. The initial optical density was set to OD600 nm $=0.1$. The cultures were grown at $37^{\circ} \mathrm{C}$ with continuous shaking at $200 \mathrm{rpm}$ for $72 \mathrm{~h}$. Next, the cultures were centrifuged $\left(14,000 \times \mathrm{g}\right.$ ) at $4{ }^{\circ} \mathrm{C}$ for $30 \mathrm{~min}$ (Sigma 6K15, 12500 rotor, DJB Labcare Ltd., Newport Pagnell, United Kingdom). The resulting supernatant was acidified to $\mathrm{pH} 2.0$ and left for $24 \mathrm{~h}$ at $4{ }^{\circ} \mathrm{C}$. The pellet was centrifuged, water added, and the acidic $\mathrm{pH}$ was neutralized with $\mathrm{NaOH}$. Then ethyl acetate was extracted and the organic fractions were collected and evaporated. The residue pellets were dissolved in ultrapure water and freeze-dried. High-performance liquid chromatographyHPLC (SM 1) was used to identify and confirm the presence of surfactin.

\subsection{Nanoemulsion Preparation}

Oil-in-water $(\mathrm{o} / \mathrm{w})$ nanoemulsions were prepared by crash dilution method [16] using CBD oil with, mixed surfactants-surfactin (S), and polyethylene glycol sorbitan monooleate $(\mathrm{P})$ and water, at the ratios presented in Table 1.

Table 1. Characteristics of the selected nanoemulsion samples.

\begin{tabular}{|c|c|c|c|c|c|c|c|c|c|c|c|}
\hline \multirow{2}{*}{ NE } & \multicolumn{4}{|c|}{$\begin{array}{l}\text { Nanoemulsion } \\
\text { Composition \% }\end{array}$} & \multicolumn{2}{|c|}{$\mathrm{D}_{\mathrm{H}}[\mathrm{nm}]$} & \multicolumn{2}{|c|}{ PdI } & \multicolumn{2}{|c|}{$\zeta[\mathrm{mV}]$} & \multirow[t]{2}{*}{$\mathrm{pH}$} \\
\hline & $S$ & $\mathbf{P}$ & O* & $\mathbf{W}$ & HIU & SON & HIU & SON & HIU & SON & \\
\hline A & 1 & 4 & 1 & 94 & $173.7 \pm 4.3$ & $333.5 \pm 6.9$ & $0.347 \pm 0.009$ & $0.450 \pm 0.018$ & $0.0 \pm 0.2$ & $-59.9 \pm 0.9$ & 3.47 \\
\hline B & 1 & 8 & 1 & 90 & $325.2 \pm 18.0$ & $497.2 \pm 17.5$ & $0.481 \pm 0.059$ & $0.633 \pm 0.026$ & $-36.7 \pm 1.2$ & $-36.9 \pm 0.7$ & 4.00 \\
\hline $\mathrm{C}$ & 8 & 4 & 1 & 87 & $254.8 \pm 22.3$ & $331.0 \pm 8.1$ & $0.347 \pm 0.029$ & $0.479 \pm 0.066$ & $-90.7 \pm 1.0$ & $-81.1 \pm 0.8$ & 6.26 \\
\hline $\mathrm{D}$ & 8 & 8 & 1 & 83 & $689.4 \pm 72$ & $1418.0 \pm 105.0$ & $0.691 \pm 0.062$ & $0,987 \pm 0.021$ & $-72.6 \pm 0.3$ & $-55.6 \pm 0.6$ & 5.64 \\
\hline $\mathrm{E}$ & 5 & 5 & 1 & 89 & $175.0 \pm 5.3$ & $621.5 \pm 20.7$ & $0.350 \pm 0.040$ & $0.625 \pm 0.075$ & $-94.7 \pm 3.9$ & $-85.1 \pm 0.1$ & 5.30 \\
\hline $\mathrm{F}$ & 10 & 10 & 1 & 80 & $222.5 \pm 2.9$ & $749.3 \pm 33.5$ & $0.355 \pm 0.046$ & $0.720 \pm 0.042$ & $-82.7 \pm 2.01$ & $-86.5 \pm 0.9$ & 6.48 \\
\hline G & 10 & 0 & 1 & 89 & $168.0 \pm 4.9$ & $586.8 \pm 32.4$ & $0.337 \pm 0.037$ & $0.658 \pm 0.017$ & $-110.3 \pm 3.5$ & $-60.2 \pm 0.1$ & 6.67 \\
\hline $\mathrm{H}$ & 20 & 0 & 1 & 79 & $880.1 \pm 21.1$ & $526.7 \pm 35.9$ & $0.829 \pm 0.149$ & $0.749 \pm 0.008$ & $-119.3 \pm 1.2$ & $-35.7 \pm 0.4$ & 6.70 \\
\hline I & 0 & 10 & 1 & 89 & $128.6 \pm 1.2$ & $216.7 \pm 4.5$ & $0.180 \pm 0.017$ & $0.409 \pm 0.016$ & $-55.5 \pm 2.7$ & $-81.7 \pm 0.2$ & 2.93 \\
\hline $\mathrm{J}$ & 0 & 20 & 1 & 79 & $133.0 \pm 2.1$ & $286.2 \pm 14.9$ & $0.363 \pm 0.008$ & $0.574 \pm 0.133$ & $-19.3 \pm 0.4$ & $-55.6 \pm 0.0$ & 3.60 \\
\hline
\end{tabular}

(S)—surfactin; (P)—polyethylene glycol sorbitan monooleate; (O)—CBD oil; $\mathrm{D}_{\mathrm{H}}$-average particle size; PdI—polydispersity index; $\zeta$ Zeta potential. * oil concentration based on manufacturer data-minimum concentration causing positive effects in topical application.

In the first step, oil, the surfactants, and water were mixed at a certain composition followed by sonication at $25^{\circ} \mathrm{C}$ for $20 \mathrm{~min}$ to form a microemulsion concentrate which was subsequently rapidly diluted four times with water resulting in nanoemulsion. The second step involved an application of a microfluidic process using an advanced Panda PLUS 2000 GEA Lab (Düsseldorf, Germany) device with appropriately established valve pressures. First on valve No. 2-150 bar, then on valve No. 1-1500 bar. After setting the parameters, the system was passed through the homogenizer three times to obtain the nanoemulsion.

\subsection{Nanoemulsion Characterization Methods}

Analysis of the physicochemical characteristics of the designed nanoemulsions involved the scattering methods such as DLS (dynamic light scattering) and electrophoretic light scattering (ELS), as well as microscopic imaging by TEM (transmission electron microscopy). 
Dynamic light scattering (DLS) and electrophoretic light scattering (ELS): the average particle size $\left(\mathrm{D}_{\mathrm{H}}\right)$ and polydispersity index $(\mathrm{PdI})$ values of the droplets were determined using DLS analysis. Zeta potential ( $($ ) evaluation was conducted using ELS. Both the DLS and ELS measurements were made using a ZetaSizer Nano ZS (Malvern Instruments, Malvern, UK) with a detection angle of $173^{\circ}$, and with a He-Ne laser $(632.8 \mathrm{~nm})$, and an ALV 5000 multibit, multitap autocorrelator (Malvern Instruments, Malvern, UK). All measurements were taken at $25{ }^{\circ} \mathrm{C}$. Each value was calculated as the average of three subsequent instrument runs with at least 20 runs.

Transmission electron microscopy (TEM): the TEM imaging was performed using a FEI Tecnai G2 XTWIN transmission electron microscope (FEI, Hillsboro, OR, USA). The nanoemulsion morphology was determined by calculating the size of approximately 250 droplets from several TEM images obtained from different sites of the grid. The samples were prepared by placing a small amount of diluted suspension on a Cu-Ni grid and stained with $2 \%$ uranyl acetate before shooting. The size distribution plots were fitted using a Gaussian curve approximation.

\subsection{Cell Culture}

The cytotoxicity effect of the nanoemulsions was evaluated against human normal dermal fibroblasts (NHDF) cell lines (Lonza, Gdańsk, Poland) and immortalized human keratinocytes (HaCaT) (LGC Standards, Łomianki, Poland). Cells between passages 6-12 were grown at $37^{\circ} \mathrm{C}$ in complete Eagle's minimum essential medium (EMEM) with alpha modification, and complete Dulbecco's modified Eagle's medium (DMEM), respectively for NHDF and $\mathrm{HaCaT}$ cell lines. Both mediums were supplemented with $10 \%$ fetal bovine serum (FBS) and glutamine $(2 \mathrm{mM})$, penicillin $(100 \mathrm{U} / \mathrm{mL})$, and streptomycin $(100 \mu \mathrm{g} / \mathrm{mL})$. Cells were grown in $175 \mathrm{~cm}^{2}$ culture flasks in a $37^{\circ} \mathrm{C}$ incubator in a humidified atmosphere containing $5 \% \mathrm{CO}_{2}$. Cells were cultivated in 96-well tissue culture plates $\left(6 \times 10^{3}\right.$ and $4 \times 10^{3}$ cells/well for NHDF and HaCaT cells, respectively) and treated with different concentrations of nanoemulsion for 24 and $48 \mathrm{~h}$. The effect of each nanoemulsion on cell viability was determined using a quantitative colorimetric MTT assay after mentioned time of treatment. Then the medium with nanoemulsions was removed and the $50 \mu \mathrm{L}$ of working MTT solution $(0.5 \mathrm{mg} / \mathrm{mL})$ was added to each well. Plates were incubated for $4 \mathrm{~h}$ at $37^{\circ} \mathrm{C}$. After the incubation, the medium was removed and $50 \mu \mathrm{L}$ of DMSO was added to dissolve the formazan crystals. The absorbance was measured at $570 \mathrm{~nm}$ on an ASYS UWM 340 microplate reader (Biogenet, Józefów, Poland). Non-treated cells were used as control. The assays were performed in triplicate and the data were expressed as mean values \pm standard deviations.

\subsection{In Vivo Skin Contact Study}

The effect of the formulation was evaluated on human skin using the NatiV3 Analyzer (Beauty of Science, Wrocław, Poland). The formulation $(1 \mathrm{~mL})$ was applied with a glass pipette and rubbed into the skin with a $1 \times 1 \mathrm{~cm}$ square matrix to determine the treated area. The nanoemulsion was applied daily, morning and evening, for 28 days. The skin was analyzed for moisture and discolorations before the start of the tests and on the 28th day of the test. The study protocol was approved by the Bioethics Commission at the Lower Silesian Chamber of Physicians and Dentists 1/PNHAB/2020 (8 July 2020).

\subsection{Statistical Analysis}

All the data are expressed as the mean values \pm standard deviation of three measurements. Statistical analyses were performed using the one-way analysis of variance with the post hoc Tukey significant difference calculator (GraphPad Prism). A value of $p<0.05$ was considered to be statistically significant. 


\section{Results}

The obtained nanosystems (Scheme 1) were formulated via a self-assembly approach to enhance penetration efficiency and bioavailability of CBD oil, as an effective system that influences skin condition. The first step of this study involved the preparation composition of spontaneous self-assembly systems. In a series of initial experiments, the optimal composition of content surfactants and operative conditions were evaluated. Duration of processing can affect the nanoemulsion stability. Becher [24] showed that the number of times the product was passed through the device, affected the mean particle size and the particle size distribution.

Multiple processing cycles caused a decrease in mean particle size and narrow particle size distribution. The efficiency of the emulsification process was recorded by measuring the mean droplet diameter. From these preliminary experiments, the homogenization was performed using three cycles of pressure 150 bar-on the second valve and 1500 bar-on the first valve, using a two-stage high-pressure homogenizer (microfluidized). The effective delivery of the solubilized CBD oil to the skin depends on the nanocarrier physicochemical properties such as particle shape, size distribution, surface charge, and morphology which were established. Visual observations and DLS analysis enable choosing forms creating the $\mathrm{o} / \mathrm{w}$ nanoemulsions, where the stabilization process of the non-ionic polyethylene glycol sorbitan monooleate was assisted by an addiction of ionic surfactin. The obtained parameters from DLS of the nanoemulsions prepared are reported in Table 1 . The results show a decrease in particle size using the high-pressure homogenization compared with the sonication method. For all systems, the results show a decrease in particle size and polydispersity index using high-pressure homogenization.

The smallest values were obtained for the nanoemulsion containing only polyethylene glycol sorbitan monooleate (samples I and J), but too low $\mathrm{pH}$ of those systems makes its skin application impossible. The CBD $(\mathrm{O})$ is a phenol derivative with easily deprotonated hydroxyl groups, but CBD is poorly soluble in water. To improve CBD solubility polyethylene glycol sorbitan monooleate $(\mathrm{P})$ is added to the mixture. As a result, samples with $\mathrm{S}$ and $\mathrm{P}$ (samples marked as I and J) and with S, P, and a little amount of surfactin (S) have acidic $\mathrm{pH}$ (samples $\mathrm{A}$ and $\mathrm{B}$ ). In the case where surfactin concentration increases the $\mathrm{pH}$ solutions also increase. Observed dependence is a result of presence in surfactin structure two deprotonated carboxylic groups $\left(-\mathrm{COO}^{-}\right)$which act as a proton acceptor. Results show the importance of the presence of surfactin in the aggregates formed. Relatively good results were obtained for combinations of equal amounts of both surfactants (sample E), but only for specific concentrations. The addition of surfactin to the formulations caused the $\mathrm{pH}$ to shift towards neutral ( $\mathrm{pH}$ 5.3-6.7), which makes them more suitable for dermal applications. Increasing the amount of surfactants showed that an increase in the number of nanodroplets (sample F) was observed. Usually, researchers consider PdI values of less than 0.5 as acceptable while 0.3 and below are regarded as optimum. If nanosystems exhibit PDI within the range of $0.1-0.4$ could be characterized as moderate polydispersity [26]. The obtained parameters were approximately 0.3 in five cases after homogenization. The obtained results clearly show the importance of homogenization in the preparation of some aggregate systems (Figure 2). It appears that the combination of mixed surfactants prevents creaming and promotes stability. Although numerous studies addressed enhancing stability by using mixed surfactants, the mechanism involved is not yet fully understood [21]. However, the importance of this concept in industrial emulsions, where it is the rule rather than the exception to use mixed surfactants, would seem to justify further research in this area. The highest $\zeta$-potential value was measured for the nanoemulsion containing the only surfactin and this value was achieved after used a homogenizer. 


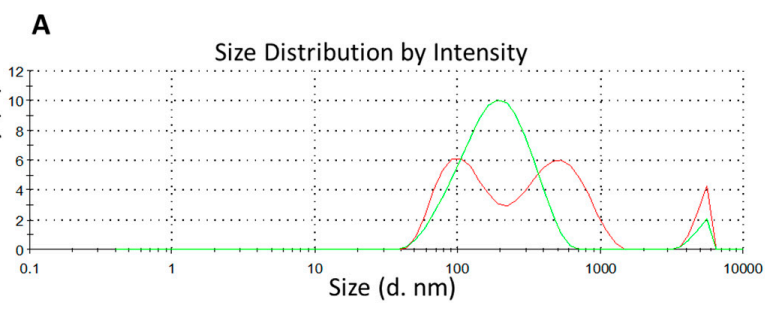

B

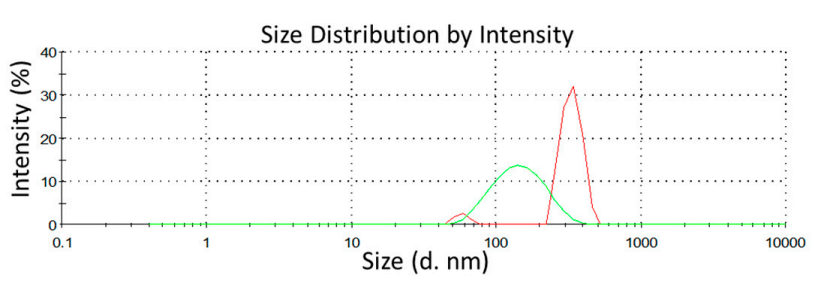

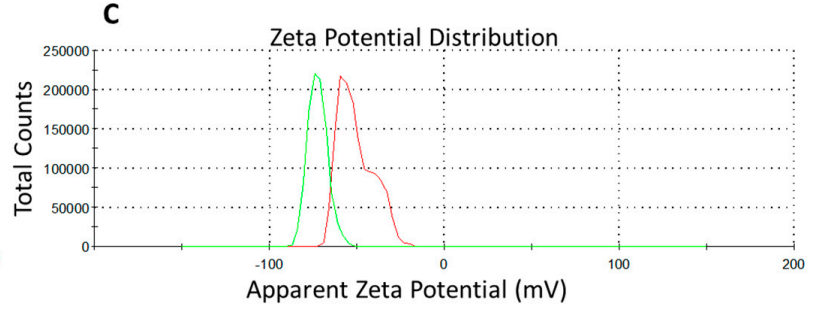

D

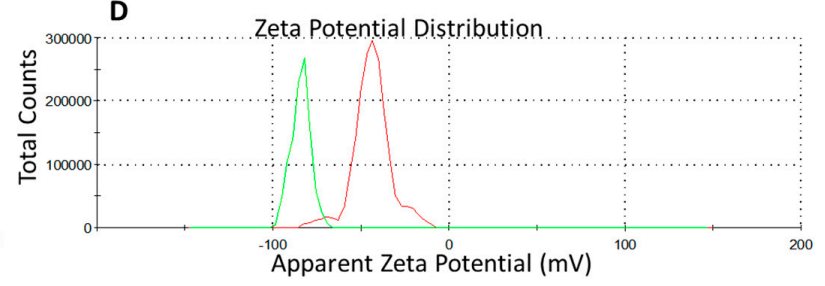

Figure 2. Characteristics of the optimized nanoemulsion systems: (A) A-NE and (B) I-NE systems along with hydrodynamic diameter $\left(\mathrm{D}_{\mathrm{H}}\right)$; (C) D-NE and (D) F-NE along with zeta potential for freshly prepared formulations: after sonication (red line) and after using microfluidizer Panda (green line).

The increase in the $\zeta$-potential may presumably be attributed to the incorporation of polar compounds, present in the surfactin, in the mixed interfacial film [19]. This interfacial film acts as a stabilizer by forming a high-energy barrier that repels adjacent droplets and leads to the formation of stabilized emulsified droplets. It is worth mentioning, however, that the CBD contribution with two hydrophilic groups can also significantly affect the $\zeta$-potential. The size of nanoemulsions was found to be consistent between the DLS and observation under TEM. The TEM images showed that droplets were spherical (Figure 3). Additionally, visual observation revealed no agglomerates, and the systems were opalescent.

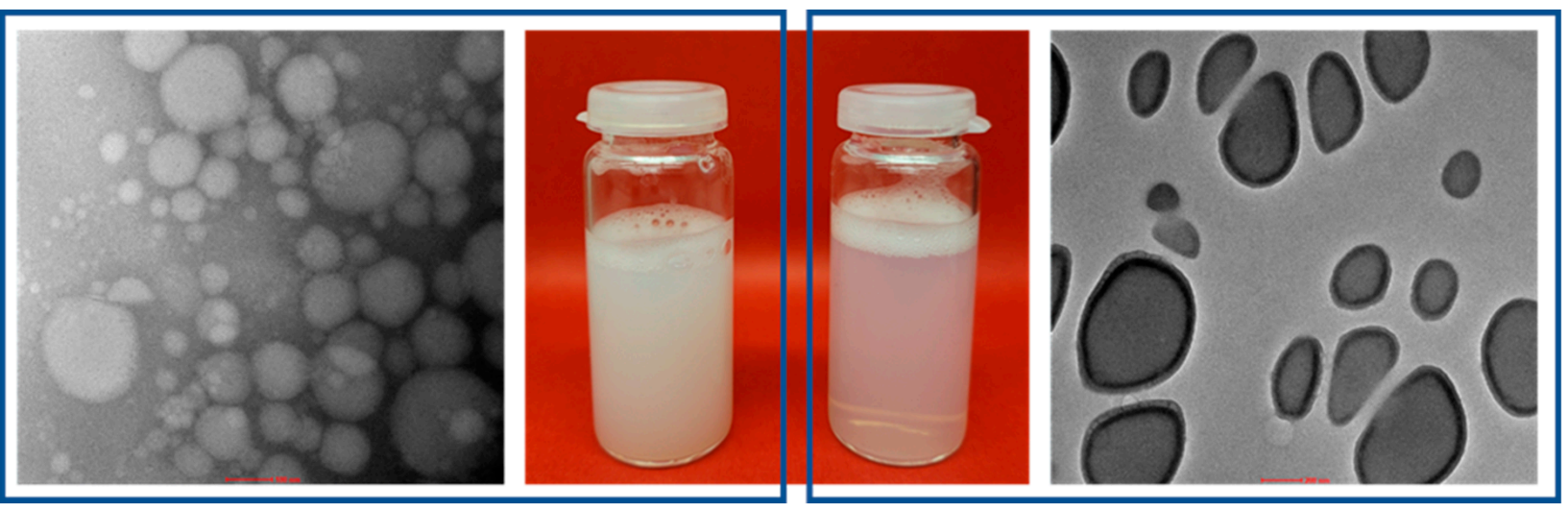

Figure 3. Transmission electron microscopy (TEM) and images of the optimized nanoemulsion F system (left after sonication, right after homogenization).

The physical stability of any nanocarrier system provides the maximum long-lasting effectiveness of the active ingredient in a given transdermal application [27]. Finally, none of the tested nanoemulsion systems showed any visual phase separation, creaming, coalescence. The stability of a nanoemulsion is one of the most important factors for a potential application. The samples were stored at $25^{\circ} \mathrm{C}$ for 30 days and their stability was evaluated based on the particle size $\left(\mathrm{D}_{\mathrm{H}}\right)$. In this case, the parameters as size remained largely unchanged during the prolonged observation period as supported by DLS measurements almost in all cases (Figure 4). It seems that an increase in polyethylene glycol sorbitan monooleate causes nanoemulsion droplets to grow after 30 days of storage. In contrast, increasing the amount of surfactin, the greater proportion, causes the droplet size 
to decrease in the majority of the preparation. The storage period had the least influence on the systems A and E. Hydrodynamic diameter slightly increased reaching $188.7 \pm 2.8$ and $195.6 \pm 12.3$ after 30 days for $A$ and E systems, respectively. Nevertheless, all results show the relative stability of the resulting systems.

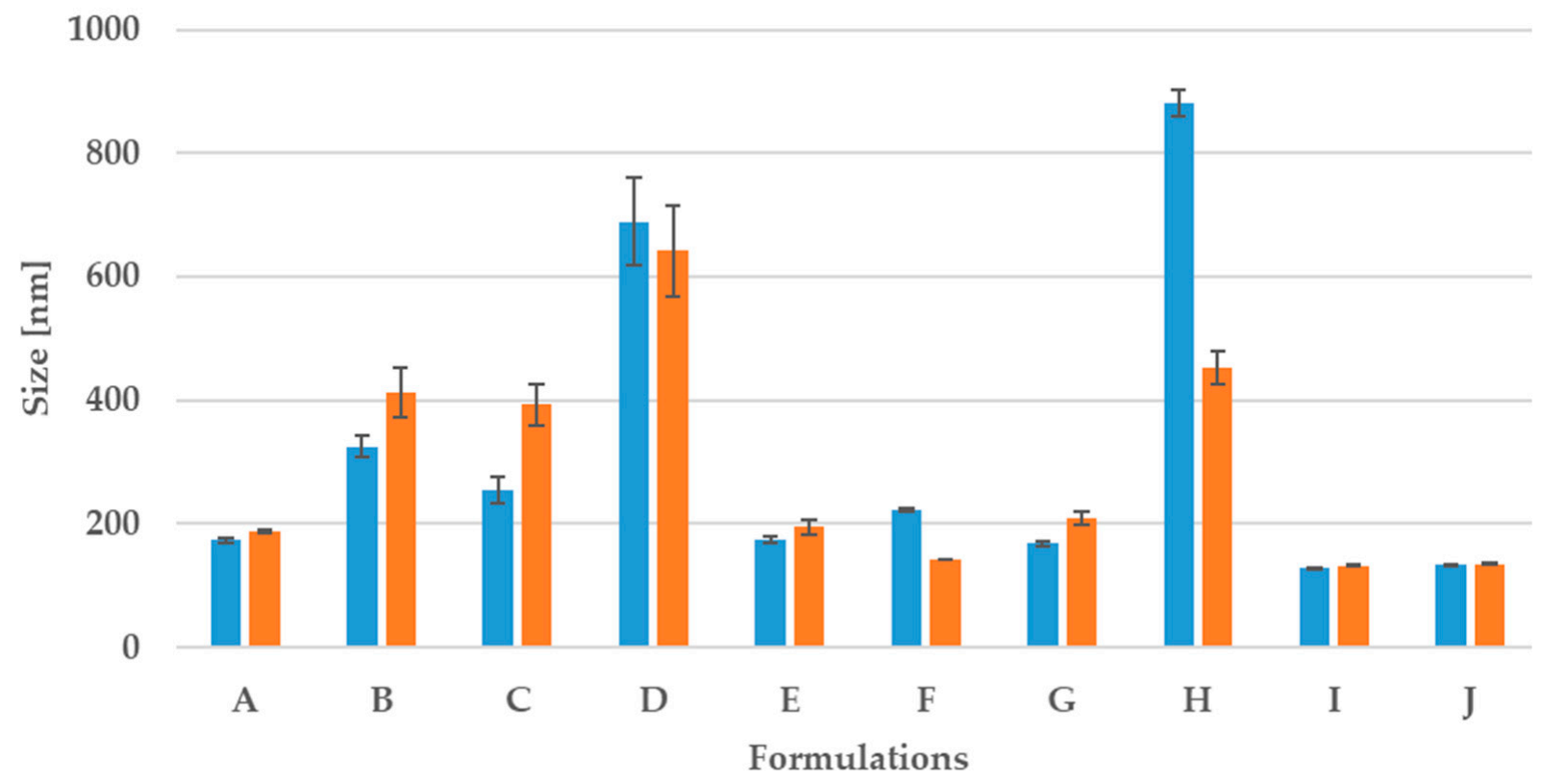

Figure 4. Changes in the size of NE during 30 days (gray after preparation) on storage at $25^{\circ} \mathrm{C}$; in blue-size after preparation, in orange-after 30 days.

\section{Biological Skin Response In Vitro and In Vivo}

The safety of the designed nanoemulsions was evaluated in skin cell cultures against normal human fibroblasts and keratinocytes. All experiments were performed in triplicate. The viability in almost all cases was over $80 \%$ after 24 and $48 \mathrm{~h}$ of exposure for diluted formulations (Figure 5). Surfactin was already successfully used in nanoformulations, which confirmed its safety upon NHDF and HaCaT cell lines. [18]. So far this is the third use of surfactin in a nanoemulsion drug delivery system [18,24]. As for Tween 80-the CIR Expert Panel evaluated different polysorbates, including Polysorbate 80, and concluded that they are safe as cosmetic ingredients [28]. Polysorbate 80 is used as an emulsifier to combine oil and water. Arechabala et al. tested various surfactants against normal human fibroblasts, and in their study, Tween 80 appeared to be the least cytotoxic [29]. It is successfully used in other nanoemulsions with no cytotoxic effect-with applications for skin $[30,31]$ and food [32]. The CBD was evaluated by Petrosino et al. in the HaCaT cell line-no effect was observed with 1, 5, 10, and $20 \mu \mathrm{M}$ of CBD after 6, 12, and $24 \mathrm{~h}$ [33]. Sangiovanni et al. obtained similar results with the same cell line in the range $0.05-5 \mu \mathrm{M}$ after 6 and $24 \mathrm{~h}$. They also checked the viability of human dermal fibroblasts (HDF) in the range from 0.1 to $2.5 \mu \mathrm{M}$ ( 6 and $24 \mathrm{~h}$ )—no cytotoxic effect in their tests either [34]. The impact of two selected nanoemulsions-E and G-was also evaluated in vivo. It has already been proven that nanoemulsions effectively penetrate agents upon skin tissue [18] and they can improve skin condition [35].

Both the formulations developed improved the condition of the skin. The use of sample E influenced discolorations, reducing them by $14.15 \%, 10.73 \%$, and $5.52 \%$, respectively, in subjects aged 30, 40, and 50 years old (Figures 6 and 7). As for the second tested nanoformulation $\mathrm{G}$, it also had a positive impact on skin decreasing discolorations by $7.66 \%, 9.49 \%$, and $3.08 \%$ in the same age subjects. 
A

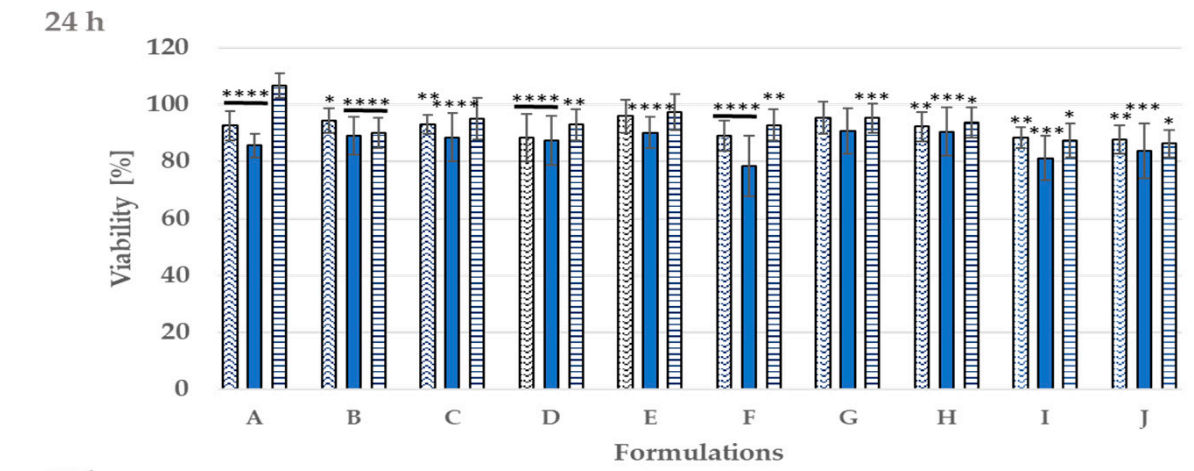

$48 \mathrm{~h}$

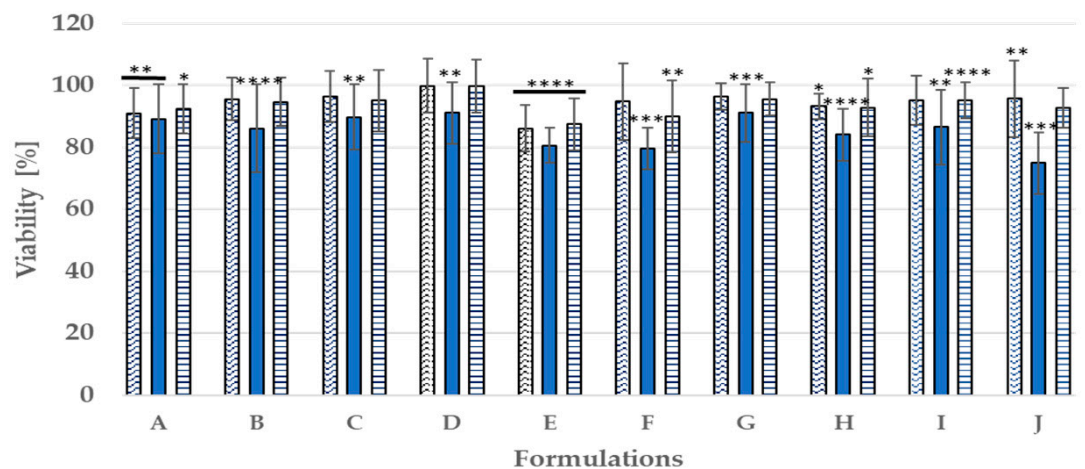

$24 \mathrm{~h}$

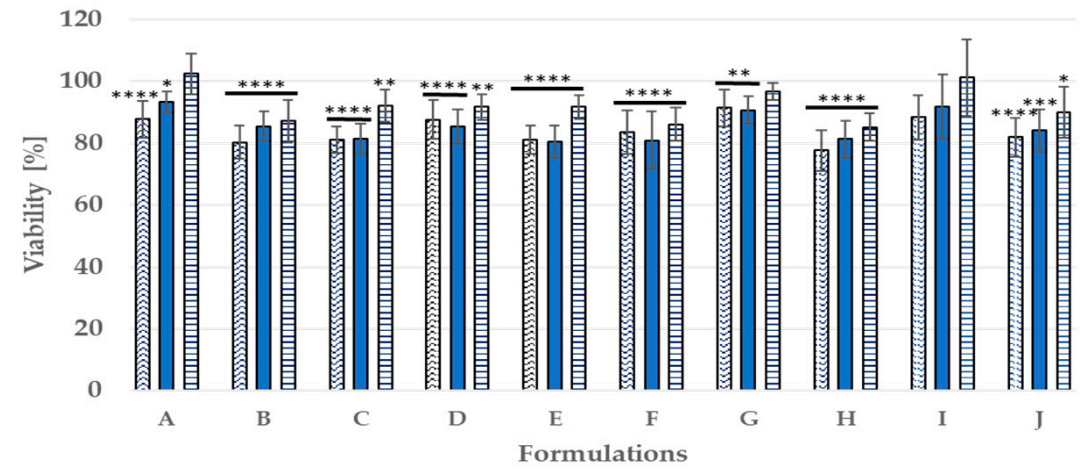

$48 \mathrm{~h}$

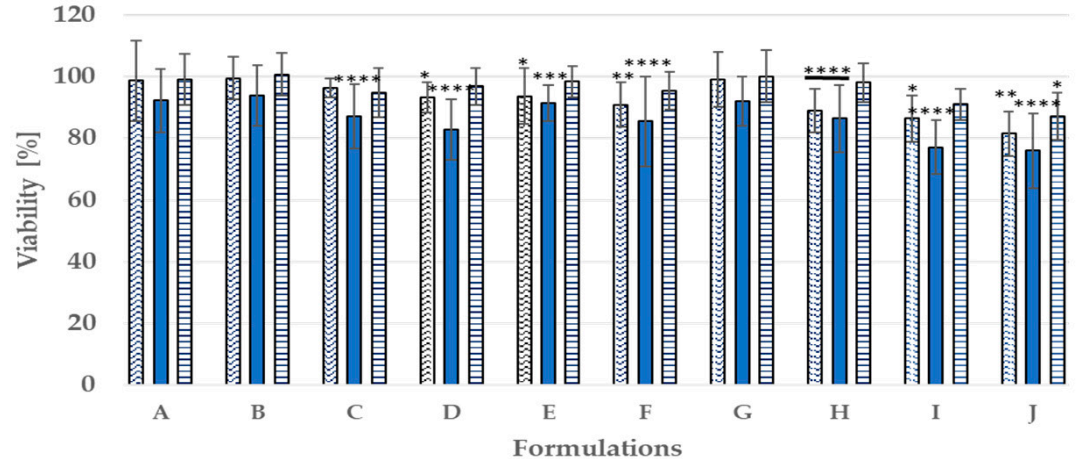

Figure 5. Cytotoxicity of the studied nanoemulsion A-H upon normal human skin cells: NHDF fibroblasts (A) and HaCaT keratinocytes (B) incubated for $24 \mathrm{~h}$ and $48 \mathrm{~h} ;{ }^{* * *} p<0.0001,{ }^{* * *} p>0.0001,{ }^{* *} p<0.001, * p<0.05$; dilutions 圌 1:50, 1:100, 
A

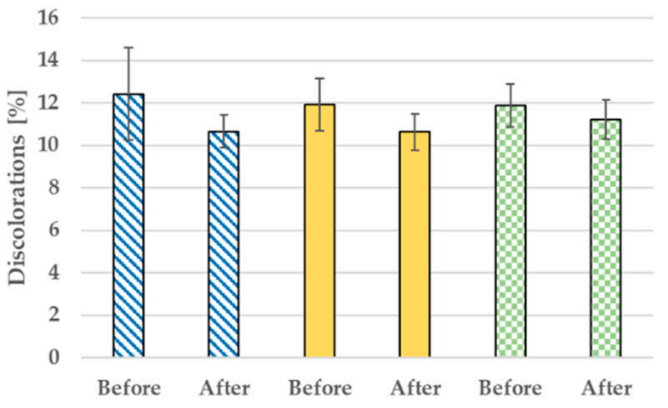

B

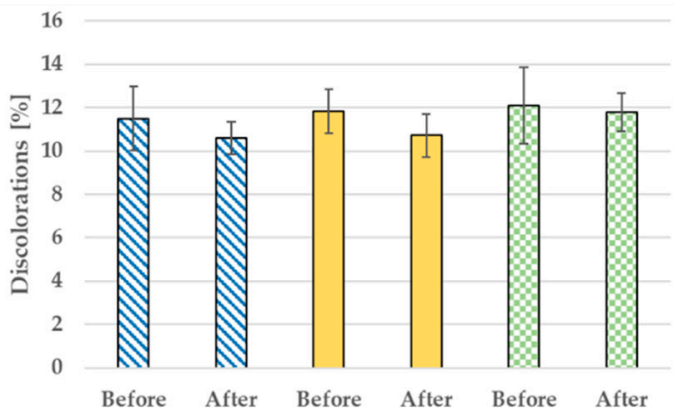

Figure 6. Discoloration reduction after 28 days of use: formulation $\mathrm{E}(\mathbf{A})$ and formulation $\mathrm{G}(\mathbf{B})$; probants' age: $\mathbb{N} 30$ s, $40 \mathrm{~s},=50 \mathrm{~s}$.

A

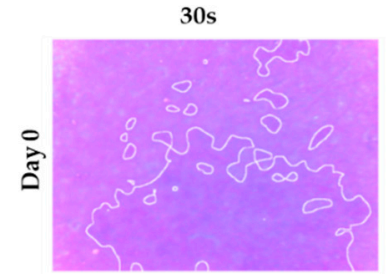

\section{Probants age}
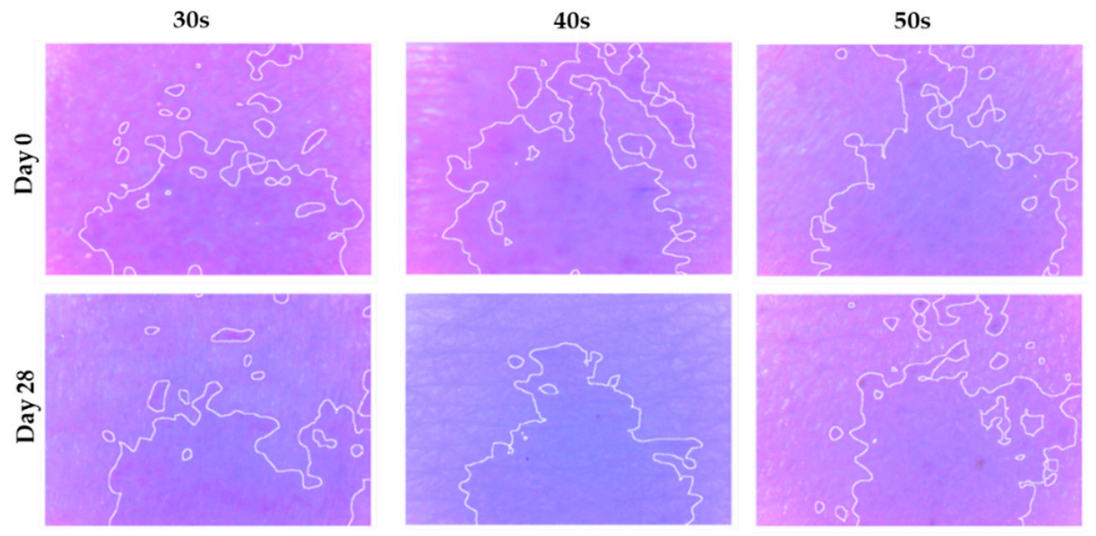

B

Probants age
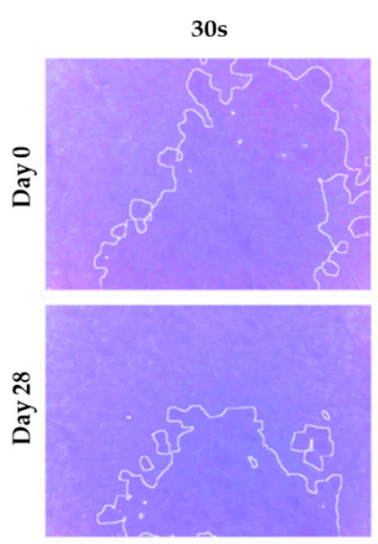

$40 \mathrm{~s}$
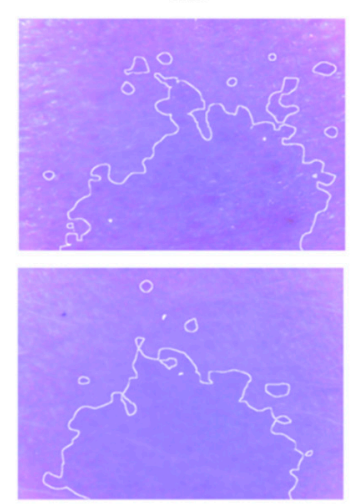
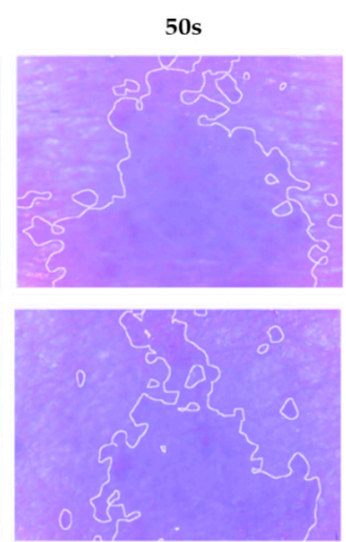

Figure 7. Selected photos showing discoloration changes before and after the application of $\mathrm{E}(\mathbf{A})$ and $\mathrm{G}$ (B) nanoemulsions.

The nanoemulsions also have a positive effect on skin moisturizing (Figure 8). In the $\mathrm{T}$ zone of the face for formulation $\mathrm{E}$, hydration increased by $33.84 \%, 22.58 \%, 44.63 \%$, while in the $U$ section is increased by $58.37 \%, 31.43 \%$, and $24.41 \%$ (Figure 6 ). In the case of $\mathrm{G}$ formulation, an improvement in skin hydration was also noted-in the $\mathrm{T}$ zone an increase of $9.09 \%, 19.37 \%$, and $29.41 \%$, and in the $U$ zone of $31.75 \%, 33.33 \%$, and $5.74 \%$. The performed experiments suggest that the nanoemulsions with CBD oil are promising formulations applicable in cosmetics. However, the presented results are preliminary so far. 
A

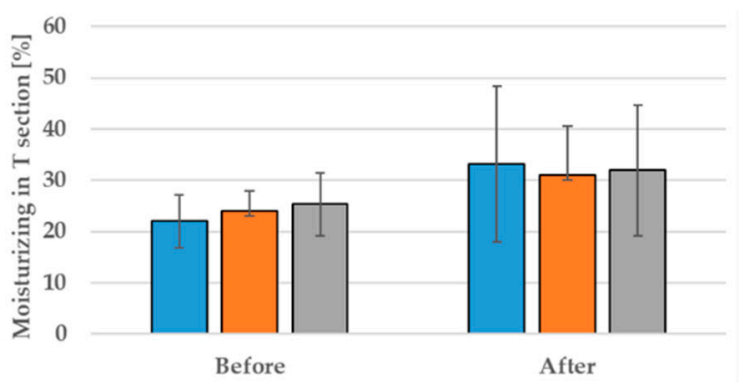

B

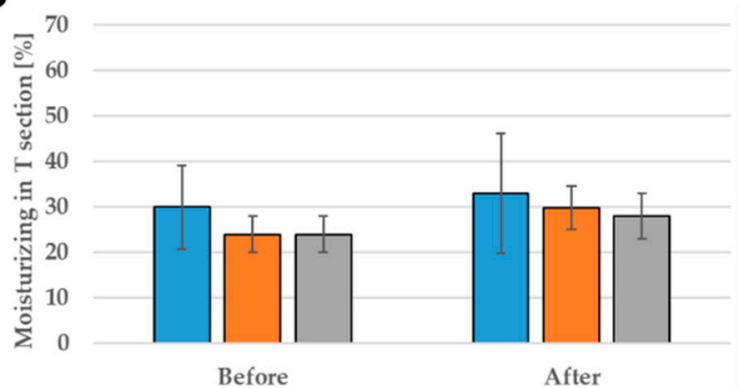

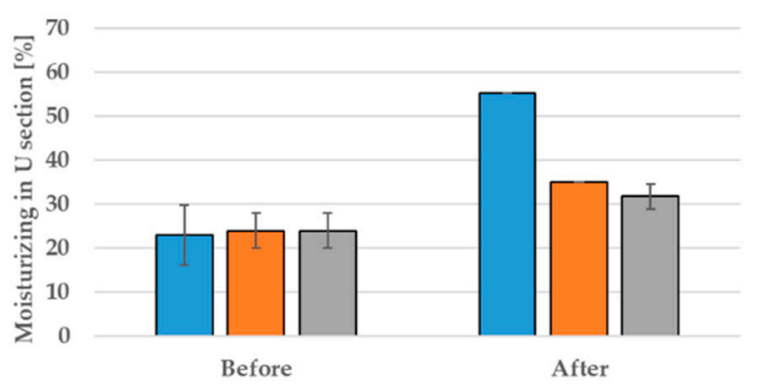

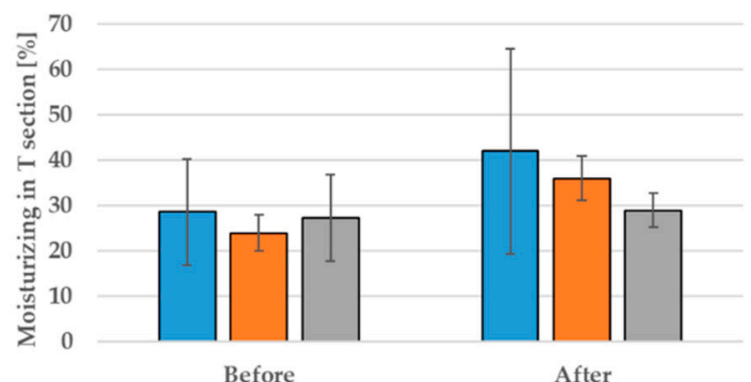

Figure 8. The moisturizing effect after 28 days of using: formulation $G$ (A) and formulation $G$ (B); probants' age: $\square$ 30s, $\square$ 40s, $\square 50$ s.

So far, there is not much data on the use of CBD nanoemulsions on the skin. Sangiovanni et al. verified Cannabis sativa L. ethanolic extract standardized in cannabidiol as an anti-inflammatory agent in the skin. The extract inhibited the release of inflammatory mediators involved in wound healing and inflammatory processes in the skin [34]. In the in vitro system represented by human keratinocytes and fibroblasts, CBD extract was found to be able to protect cells against cytotoxicity and apoptosis induced by oxidative stress [36]. Hence, these data suggest a possible positive effect on the skin after using $\mathrm{CBD}$ nanoemulsions. Further research is required for the use of $\mathrm{CBD}$ in nano-formulations intended for application to the skin.

\section{Conclusions}

Nanoemulsions were prepared with the use of a mixture of surfactants, i.e., ionic surfactin and non-ionic polyethylene glycol sorbitan monooleate to stabilize oil-in-water nanoemulsion systems. The two-step process design showed significant differences in the obtained parameters characterizing the obtained formulations. The sonication method led to the obtained much larger droplets. The use of high-pressure homogenization (microfluidization) reduced their hydrodynamic radius and polydispersity index almost by half. Additionally, negative ionic surfactin positively influenced the Zeta potential. Transmission electron microscopy imaging showed the nearly spherical shape of the particle structure with a size below $200 \mathrm{~nm}$, for the best systems obtained by the microfluidization approach. The tested systems showed stability within 30 days after fabrication without loss of particle size consistency. Further studies were extended for the biocompatibility investigation using human skin cell lines HaCaT keratinocytes and NHDF normal human dermal fibroblasts, which showed a lack of toxicity towards the cells in a broad range of concentrations. The use of the NatiV3 analyzer made it possible to state that CBD oil enclosed in the nanoemulsion oleic core has a positive effect on the parameters of human skin such as hydration and degree of discoloration.

Funding: Financial support from the statutory activity of subsidy from the Polish Ministry of Science and Higher Education for the Faculty of Chemistry of the University of Wroclaw is gratefully acknowledged. Work partially financed by: POIR.01.01.01-00-0729/19. 
Institutional Review Board Statement: The study was conducted according to the guidelines of the Declaration of Helsinki and approved by the Bioethics Commission at the Lower Silesian Chamber of Physicians and Dentists 1/PNHAB/2020 (8 July 2020).

Informed Consent Statement: Informed consent was obtained from all subjects involved in the study.

Acknowledgments: The author would like to thank and acknowledge Marta Domżał-Kędzia, Department of Biotransformation, Faculty of Biotechnology, University of Wroclaw, for her invaluable technical assistance with the cell culture.

Conflicts of Interest: The author declares no conflict of interest.

\section{References}

1. Morales, P.; Reggio, P.H. CBD: A New Hope. ACS Med. Chem. Lett. 2019, 694-695. [CrossRef]

2. McPartland, J.M.; Duncan, M.; Di Marzo, V.; Pertwee, R.G. Are cannabidiol and $\Delta$ 9-tetrahydrocannabivarin negative modulators of the endocannabinoid system? A systematic review. Br. J. Pharmacol. 2015, 172, 737-753. [CrossRef] [PubMed]

3. Duran, M.; Pérez, E.; Abanades, S.; Vidal, X.; Saura, C.; Majem, M.; Arriola, E.; Rabanal, M.; Pastor, A.; Farré, M.; et al. Preliminary efficacy and safety of an oromucosal standardized cannabis extract in chemotherapy-induced nausea and vomiting. $B r$. $J$. Clin. Pharmacol. 2010, 70, 656-663. [CrossRef]

4. Pisanti, S.; Malfitano, A.M.; Ciaglia, E.; Lamberti, A.; Ranieri, R.; Cuomo, G.; Abate, M.; Faggiana, G.; Proto, M.C.; Fiore, D.; et al. Cannabidiol: State of the art and new challenges for therapeutic applications. Pharmacol. Ther. 2017, 175, 133-150. [CrossRef] [PubMed]

5. Crippa, J.A.S.; Nogueira Derenusson, G.; Borduqui Ferrari, T.; Wichert-Ana, L.; Duran, F.L.S.; Martin-Santos, R.; Vinícius Simões, M.; Bhattacharyya, S.; Fusar-Poli, P.; Atakan, Z.; et al. Neural basis of anxiolytic effects of cannabidiol (CBD) in generalized social anxiety disorder: A preliminary report. J. Psychopharmacol. 2011, 25, 121-130. [CrossRef] [PubMed]

6. Casares, L.; García, V.; Garrido-Rodríguez, M.; Millán, E.; Collado, J.A.; García-Martín, A.; Peñarando, J.; Calzado, M.A.; de la Vega, L.; Muñoz, E. Cannabidiol induces antioxidant pathways in keratinocytes by targeting BACH1. Redox Biol. 2020, $28,101321$. [CrossRef] [PubMed]

7. Al Jourdi, H.; Popescu, C.; Udeanu, D.I.; Arsene, A.; Sevastre, A.N.I.; Velescu, B. Ștefan; Lupuliasa, D. Comparative study regarding the physico-chemical properties and microbiological activities of olea europaea l. Oil and cannabis sativa 1 . seed oil obtained by cold pressing. Farmacia 2019, 67, 759-763. [CrossRef]

8. Gegotek, A.; Biernacki, M.; Ambrozewicz, E.; Surazyński, A.; Wroński, A.; Skrzydlewska, E. The cross-talk between electrophiles, antioxidant defence and the endocannabinoid system in fibroblasts and keratinocytes after UVA and UVB irradiation. J. Dermatol. Sci. 2016, 81, 107-117. [CrossRef]

9. Ständer, S.; Schmelz, M.; Metze, D.; Luger, T.; Rukwied, R. Distribution of cannabinoid receptor 1 (CB1) and 2 (CB2) on sensory nerve fibers and adnexal structures in human skin. J. Dermatol. Sci. 2005, 38, 177-188. [CrossRef]

10. Pucci, M.; Pasquariello, N.; Battista, N.; Di Tommaso, M.; Rapino, C.; Fezza, F.; Zuccolo, M.; Jourdain, R.; Agrò, A.F.; Breton, L.; et al. Endocannabinoids stimulate human melanogenesis via type-1 cannabinoid receptor. J. Biol. Chem. 2012, 287, 15466-15478. [CrossRef]

11. Baswan, S.M.; Klosner, A.E.; Glynn, K.; Rajgopal, A.; Malik, K.; Yim, S.; Stern, N. Therapeutic potential of cannabidiol (CBD) for skin health and disorders. Clin. Cosmet. Investig. Dermatol. 2020, 13, 927-942. [CrossRef]

12. Oláh, A.; Tóth, B.I.; Borbíró, I.; Sugawara, K.; Szöllõsi, A.G.; Czifra, G.; Pál, B.; Ambrus, L.; Kloepper, J.; Camera, E.; et al. Cannabidiol exerts sebostatic and antiinflammatory effects on human sebocytes. J. Clin. Investig. 2014, 124, 3713-3724. [CrossRef] [PubMed]

13. Andre, C.M.; Hausman, J.F.; Guerriero, G. Cannabis sativa: The plant of the thousand and one molecules. Front. Plant Sci. 2016, 7, 1-17. [CrossRef]

14. Tóth, K.F.; Ádám, D.; Bíró, T.; Oláh, A. Cannabinoid signaling in the skin: Therapeutic potential of the "c(ut)annabinoid" system. Molecules 2019, 24, 918. [CrossRef] [PubMed]

15. Liguori, I.; Russo, G.; Curcio, F.; Bulli, G.; Aran, L.; Della-Morte, D.; Gargiulo, G.; Testa, G.; Cacciatore, F.; Bonaduce, D.; et al. Oxidative stress, aging, and diseases. Clin. Interv. Aging 2018, 13, 757-772. [CrossRef]

16. Lewińska, A.; Jaromin, A.; Jezierska, J. Role of architecture of N-oxide surfactants in the design of nanoemulsions for Candida skin infection. Colloids Surf. B Biointerfaces 2019, 187. [CrossRef] [PubMed]

17. Fandzloch, M.; Jaromin, A.; Zaremba-Czogalla, M.; Wojtczak, A.; Lewińska, A.; Sitkowski, J.; Wiśniewska, J.; Łakomska, I.; Gubernator, J. Nanoencapsulation of a ruthenium(ii) complex with triazolopyrimidine in liposomes as a tool for improving its anticancer activity against melanoma cell lines. Dalt. Trans. 2020, 49, 1207-1219. [CrossRef]

18. Lewińska, A.; Domżał-Kędzia, M.; Jaromin, A.; Łukaszewicz, M. Nanoemulsion stabilized by safe surfactin from Bacillus subtilis as a multifunctional, custom-designed smart delivery system. Pharmaceutics 2020, 12, 953. [CrossRef]

19. Peng, L.C.; Liu, C.H.; Kwan, C.C.; Huang, K.F. Optimization of water-in-oil nanoemulsions by mixed surfactants. Colloids Surf. A Physicochem. Eng. Asp. 2010, 370, 136-142. [CrossRef]

20. Cho, Y.H.; Kim, S.; Bae, E.K.; Mok, C.K.; Park, J. Formulation of a cosurfactant-free O/W microemulsion using nonionic surfactant mixtures. J. Food Sci. 2008, 73, 115-121. [CrossRef] 
21. Reitermayer, D.; Kafka, T.A.; Lenz, C.A.; Vogel, R.F. Interrelation between Tween and the membrane properties and high pressure tolerance of Lactobacillus plantarum. BMC Microbiol. 2018, 18, 1-14. [CrossRef] [PubMed]

22. Seydlová, G.; Svobodová, J. Review of surfactin chemical properties and the potential biomedical applications. Cent. Eur. J. Med. 2008, 3, 123-133. [CrossRef]

23. Wu, Y.S.; Ngai, S.C.; Goh, B.H.; Chan, K.G.; Lee, L.H.; Chuah, L.H.; Vecino, X.; Cruz, J.M.; Moldes, A.B.; Rodrigues, L.R.; et al. Anticancer activities of surfactin potential application of nanotechnology assisted surfactin delivery. Front. Pharmacol. 2017, 21, 423-433. [CrossRef] [PubMed]

24. Ganesan, N.G.; Rangarajan, V. A kinetics study on surfactin production from Bacillus subtilis MTCC 2415 for application in green cosmetics. Biocatal. Agric. Biotechnol. 2021, 33. [CrossRef]

25. Joe, M.M.; Bradeeba, K.; Parthasarathi, R.; Sivakumaar, P.K.; Chauhan, P.S.; Tipayno, S.; Benson, A.; Sa, T. Development of surfactin based nanoemulsion formulation from selected cooking oils: Evaluation for antimicrobial activity against selected food associated microorganisms. J. Taiwan Inst. Chem. Eng. 2012, 43, 172-180. [CrossRef]

26. Campelo, M.d.S.; Melo, E.O.; Arrais, S.P.; do Nascimento, F.B.S.A.; Gramosa, N.V.; Soares, S.d.A.; Ribeiro, M.E.N.P.; da Silva, C.R.; Júnior, H.V.N.; Ricardo, N.M.P.S. Clove essential oil encapsulated on nanocarrier based on polysaccharide: A strategy for the treatment of vaginal candidiasis. Colloids Surf. A Physicochem. Eng. Asp. 2021, 610. [CrossRef]

27. Raju, N.S.; Krishnaswami, V.; Vijayaraghavalu, S.; Kandasamy, R. Nanocosmetics, Fundamentals, Applications and Toxicity, Micro and Nano Technologies. In Transdermal and Bioactive Nanocarriers; Elsevier: Amsterdam, The Netherlands, 2020; pp. 17-33. [CrossRef]

28. Bergfeld, W.F.; Donald, V.; Hill, R.A.; Klaassen, C.D.; Liebler, D.C.; Marks, J.G.; Shank, C.; Slaga, T.J.; Snyder, P.W. Safety Assessment of Polysorbates as Used in Cosmetics. Cosmet. Ingred. Rev. 2015. Available online: https://www.cir-safety.org/sites/ default/files/polysorbates_0.pdf (accessed on 22 May 2015).

29. Arechabala, B.; Coiffard, C.; Rivalland, P.; Coiffard, L.J.M.; De Roeck-Holtzhauer, Y. Comparison of cytotoxicity of various surfactants tested on normal human fibroblast cultures using the neutral red test, MTT assay and LDH release. J. Appl. Toxicol. 1999, 19, 163-165. [CrossRef]

30. Md Saari, N.H.; Chua, L.S.; Hasham, R.; Yuliati, L. Curcumin-loaded nanoemulsion for better cellular permeation. Sci. Pharm. 2020, 88, 44. [CrossRef]

31. Teo, S.Y.; Yew, M.Y.; Lee, S.Y.; Rathbone, M.J.; Gan, S.N.; Coombes, A.G.A. In Vitro Evaluation of Novel Phenytoin-Loaded Alkyd Nanoemulsions Designed for Application in Topical Wound Healing. J. Pharm. Sci. 2017, 106, 377-384. [CrossRef]

32. Wooster, T.J.; Moore, S.C.; Chen, W.; Andrews, H.; Addepalli, R.; Seymour, R.B.; Osborne, S.A. Biological fate of food nanoemulsions and the nutrients they carry-internalisation, transport and cytotoxicity of edible nanoemulsions in Caco-2 intestinal cells. RSC Adv. 2017, 7, 40053-40066. [CrossRef]

33. Petrosino, S.; Verde, R.; Vaia, M.; Allará, M.; Iuvone, T.; Di Marzo, V. Anti-inflammatory properties of cannabidiol, a nonpsychotropic cannabinoid, in experimental allergic contact dermatitis. J. Pharmacol. Exp. Ther. 2018, 365, 652-663. [CrossRef] [PubMed]

34. Sangiovanni, E.; Fumagalli, M.; Pacchetti, B.; Piazza, S.; Magnavacca, A.; Khalilpour, S.; Melzi, G.; Martinelli, G.; Dell'Agli, M. Cannabis sativa L. extract and cannabidiol inhibit in vitro mediators of skin inflammation and wound injury. Phyther. Res. 2019, 33, 2083-2093. [CrossRef] [PubMed]

35. Lewińska, A.; Domżał-Kędzia, M.; Kierul, K.; Bochynek, M.; Pannert, D.; Nowaczyk, P.; Łukaszewicz, M. Targeted Hybrid Nanocarriers as a System Enhancing the Skin Structure. Molecules 2021, 26, 1063. [CrossRef] [PubMed]

36. Di Giacomo, V.; Recinella, L.; Chiavaroli, A.; Orlando, G.; Cataldi, A.; Rapino, M.; Di Valerio, V.; Politi, M.; Antolini, M.D.; Acquaviva, A.; et al. Metabolomic profile and antioxidant/anti-inflammatory effects of industrial hemp water extract in fibroblasts, keratinocytes and isolated mouse skin specimens. Antioxidants 2021, 10, 44. [CrossRef] [PubMed] 